\title{
Bilateral Emphysematous Pyelonephritis
}

\author{
Wg Cdr Ashima Vaidya*, Sqn Ldr Mukul Bhatia ${ }^{+}$
}

MJAFI 2005; 61 : 393-394

Key Words: Urinary tract infections; Diabetes; Emphysematous Pyelitis; Emphysematous Pyelonephritis

\section{Introduction}

Es mphysematous or gas forming infections, a very small percentage of bacterial infections of the urinary tract attract importance because of their life threatening potential. The spectrum of gas producing infections include emphysematous pyelonephritis, emphysematous pyelitis and emphysematous cystitis. The first case of 'pneumaturia' was reported in 1898 [1] and since then more than 200 cases of emphysematous pyelonephritis have been reported. Bilateral emphysematous pyelonephritis is much rarer infection with fewer than $10 \%$ emphysematous pyelonephritis. More than $90 \%$ of emphysematous pyelonephritis occurs in patients with poorly controlled diabetes [2]. This report represents this rare infection.

\section{Case Report}

The patient was a 55 year old lady, known case of Type 2 diabetes mellitus, on oral hypoglycaemic agents for the past ten years with poor diabetic control. She had fever, nausea, generalized body ache and dysuria for 2 weeks. Laboratory investigation revealed anemia, azotemia with a blood urea of $100 \mathrm{mg} \%$ and a serum creatinine of $3.2 \mathrm{mg} \%$. Urine culture grew E Coli. Ultrasound of the abdomen revealed highamplitude flat anterior margin echoes within the pelvicalyceal

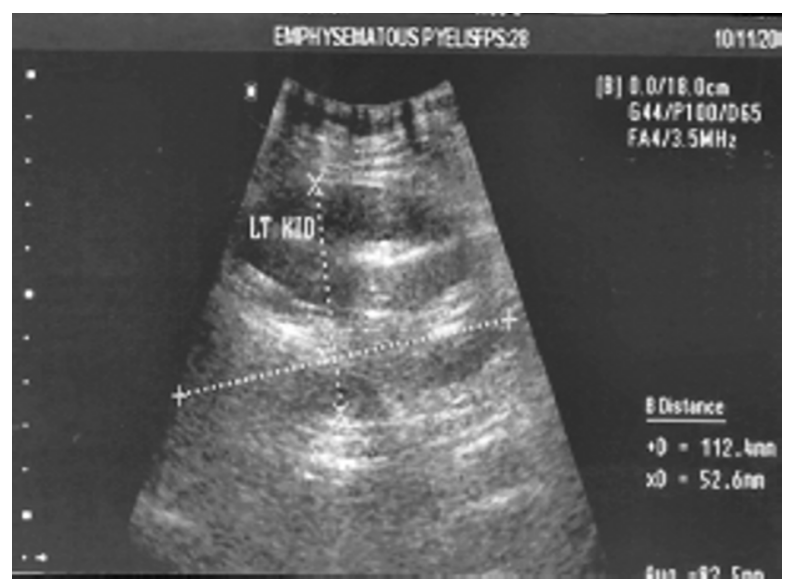

Fig. 1: Ultrasound scan of the left kidney showing echogenic foci within the kidney associated with 'dirty' distal shadowing system associated with distal shadowing of low level echoes and reverberations bilaterally (Fig 1 and 2). KUB radiograph revealed gas within the urinary collecting system bilaterally (Fig 3). An air pyleogram was noted on the left side. The patient's diabetic state was controlled with insulin and she was started on parenteral antibiotics. In view of the azotemia and bilateral presentation she was sent to an urology center for further management.

\section{Discussion}

Emphysematous pyelonephritis (EPN) is an uncommon potentially life-threatening condition characterized by the production of gas within the renal parenchyma, collecting system or the perirenal tissues $[3,4]$. The spectrum of gas producing infections includes EPN, emphysematous pyelitis (EP) and emphysematous cystitis. EPN are renal tract infections with intraparenchymal renal gas. Gas confined to the renal pelvis is EP. EPN should be regarded as a severe necrotizing form of pyelonephritis. Bilateral involvement of the kidneys is extremely rare with less than 25 reported cases in literature [5].

There are two types of EPN based on radiological findings [3]. Type I is characterized by parenchymal destruction and the presence of streaky or mottled

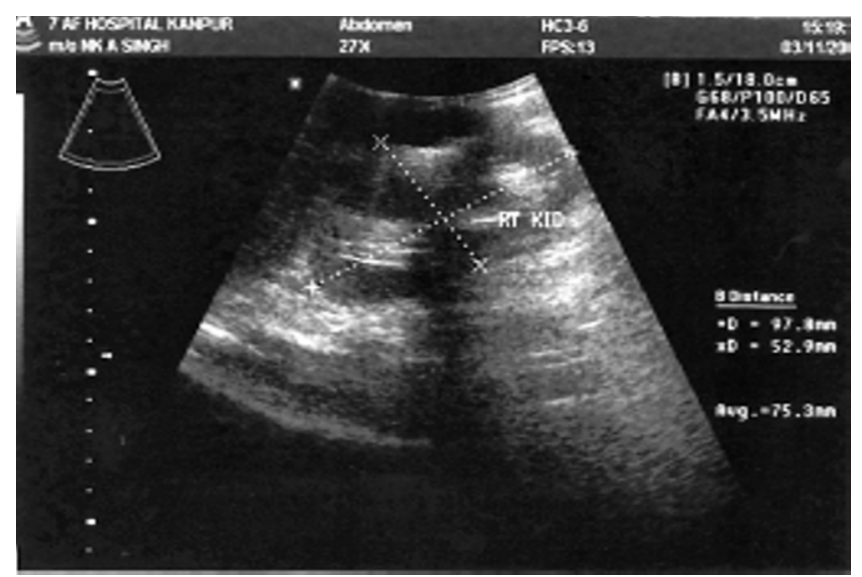

Fig. 2 : Ultrasound scan of the right kidney showing echogenic foci within the kidney associated with 'dirty' distal shadowing 


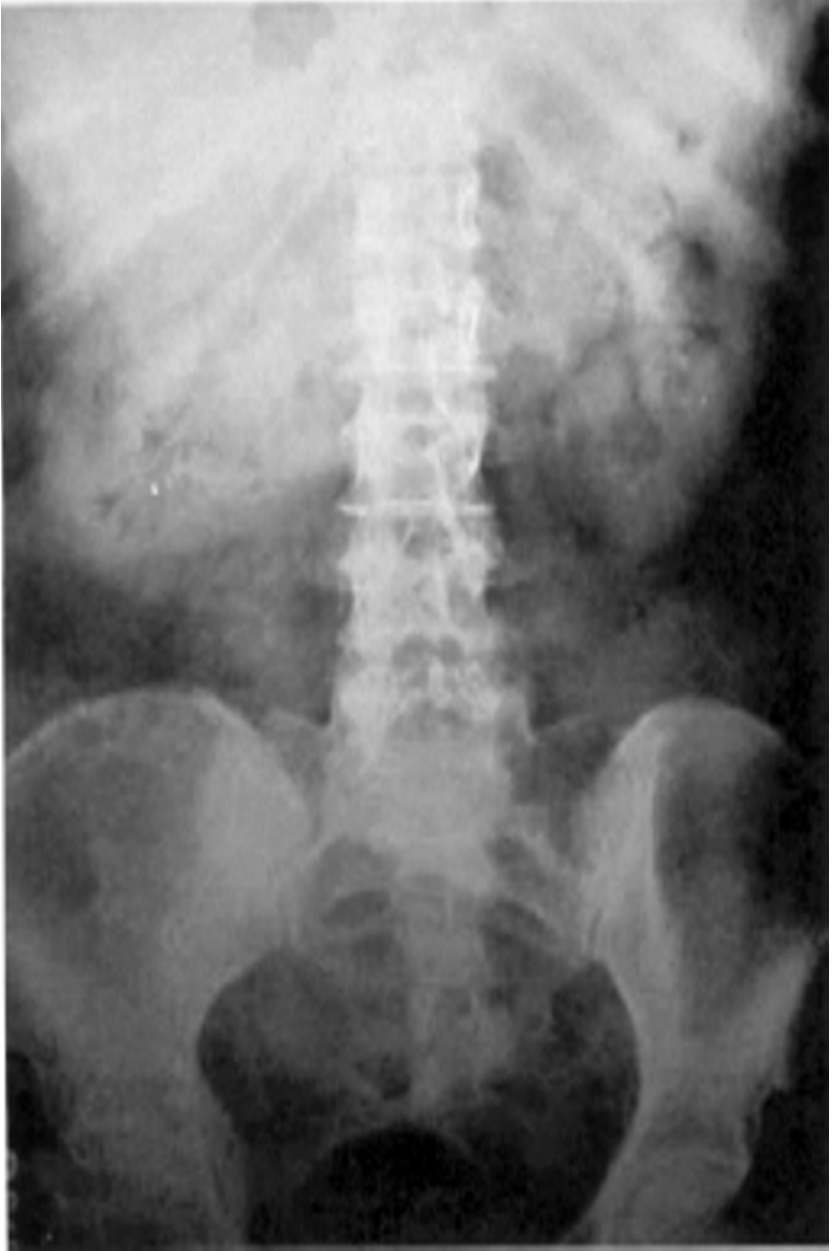

Fig. 3 : Radiograph of KUB region showing air lucencies overlying the renal areas with an 'air pyelogram' on the left side

appearing gas and absence of renal and perirenal fluid collection. Type II is characterized by renal or perirenal fluid collection with a bubbly or loculated gas within the collecting system. Type I represents classic EPN which has high mortality (69\%). Gas within the renal pelvis and ureter was found only in patients with type II EPN, in which the prognosis is favourable with a mortality rate of $18 \%$. More than $90 \%$ of all EPN occur in patients with diabetes mellitus, often uncontrolled. Rare cases have been reported in persons with renal failure and immunosuppression acting as contributing factor. EP represents gas-forming urinary tract infection that is associated with gas localized to the collecting system. It has a better prognosis compared to EPN and has not been reported frequently in radiology literature [3].

E Coli is the most common infecting organism with EPN (70\%) followed by Klebsiella, Proteus, Pseudomonas and Streptococcus. Mixed organisms are found in $10 \%$ cases. The cause of gas formation (carbon dioxide, nitrogen and hydrogen) is not well understood and appears to be due to acid fermentation of glucose rich substrate in patients with severe necrotizing infections [6].

The diagnosis of emphysematous infections is delayed as clinical findings may be nonspecific and similar to uncomplicated pyelonephritis. The sensitivity of radiography is low (33\%). Air pyelogram may be obscured by overlying loops of bowel. Ultrasound appearance is that of high amplitude flat anterior margin echoes within the renal sinus or calyces, associated with distal shadowing described as 'dirty' and associated with low-level echoes and reverberations. In theory, this shadowing can be differentiated from echo-free acoustic shadowing associated with renal calculi [5]. In practice however, ultrasound images may be interpreted variably. CT remains the modality of choice for diagnosing this condition and for staging. Ureteric stones are precisely evaluated and differentiated from intracalyceal, intrapelvic or perinephric gas collections.

The prognosis and clinical management of EP and EPN are different. As opposed to EPN, EP is a benign condition [3]. Most cases of EP respond to medical therapy consisiting of fluid resuscitation, control of diabetes and treatment with systemic antibiotics. If imaging reveals obstruction it can be relieved by percutaneous drainage or stent placement. Nephrectomy is the treatment of choice for most patients with EPN. However, the less invasive option of percutaneous drainage is increasingly used in patients as nephrectomy is associated with substantial mortality.

\section{References}

1. Kelly HA, MacCullum WG: Pneumaturia. JAMA 1898; 31: 375-81.

2. Evanroff GV, Thompson CS, Foley R, Weinman EJ. Spectrum of gas within the kidney. Emphysematous pyelonephritis and emphysematous pyelitis. Am J Med 1987; 83: 149-54.

3. Catherine Roy. Empohysematous Pyelitis: Findings in Five Patients. Radiology 2001; 218: 647-50.

4. Lee TY. Acute gas producing bacterial renal infections: correlation between imaging findings and clinical outcome. Radiology 1996; 198: 433-8.

5. Stein JP, Spitz A. Bilateral emphysematous pyelonephritis: a case report and review of the literature. Urology 1996 Jan; 47(1): 129-34.

6. Jeng H. Mixed acid fermentation of glucose as a mechanism of emphysematous urinary tract infection. J Urol 1990; 146: 146-51. 